\title{
Protective effects of paeoniflorin against FasL-induced apoptosis of intervertebral disc annulus fibrosus cells via Fas-FasL signalling pathway
}

\author{
SHAO-QING CHEN ${ }^{1}$, JIAN-PING LIN ${ }^{2}$, QI-KAI ZHENG ${ }^{2}$, SHUI-JIN CHEN ${ }^{2}$, \\ MING LI ${ }^{1}$, XIAN-ZHAO LIN ${ }^{3}$ and SHI-ZHONG WANG ${ }^{2}$ \\ ${ }^{1}$ Department of Rehabilitation Medicine, Fujian University of Traditional Chinese Medicine, Fuzhou, Fujian 350122; \\ ${ }^{2}$ The Affiliated Rehabilitation Hospital to Fujian University of Traditional Chinese Medicine; \\ ${ }^{3}$ Ministry of Education (Provincial Total Construction) Key Laboratory of Chinese \\ Orthopedics and Athletic Rehabilitation, Fuzhou, Fujian 350000, P.R. China
}

Received April 10, 2014; Accepted December 29, 2014

DOI: $10.3892 /$ etm.2015.2776

\begin{abstract}
In the present study, we demonstrate that the degeneration of intervertebral discs is caused by ageing and apoptosis of matrix cells. Apoptosis is as essential as the function of proteoglycan synthesis in assessing the possible degeneration of intervertebral discs; paeoniflorin (PF) induces cytoprotective effects on various types of cells. In this study, the function of PF in inhibiting Fas ligand (FasL)-induced apoptosis in annulus fibrosus cells was assessed, and the correlation between apoptosis and the Fas-FasL pathway was determined. Annulus fibrosus cells were derived from the intervertebral discs of 1-month-old Sprague Dawley rats; the cells were characterised by toluidine blue staining and subjected to apoptosis with FasL. PF was diluted to various concentrations and added to annulus fibrosus cells at various times. The impact of PF and FasL on cell apoptosis of annulus fibrosus cells was determined by flow cytometry. Western blot analysis was performed to determine the protein expression levels of Fas and caspase-3. The percentages of apoptotic annulus fibrosus cells as well as the expression levels of caspase-3 and Fas were significantly reduced following treatment with $208,20.8$ or $2.08 \mu \mathrm{M}$ PF. PF inhibits the activation of the Fas-FasL signal pathway and decreases FasL-induced apoptosis of annulus fibrosus cells.
\end{abstract}

Correspondence to: Professor Shi-Zhong Wang, The Affiliated Rehabilitation Hospital to Fujian University of Traditional Chinese Medicine, 282 Wusi Road, Fuzhou, Fujian 350000, P.R. China E-mail: shizhongwangcn@126.com

Key words: paeoniflorin, apoptosis, intervertebral disc annulus fibrosus cells

\section{Introduction}

The intervertebral discs are degenerated due to ageing and the apoptosis of matrix cells. Imbalances between apoptosis and regeneration cause the regression of the intervertebral disc (1). Apoptosis is as essential as the function of proteoglycan synthesis in determining the possible degeneration of intervertebral discs. As early as 1982, nucleus pulposus was observed with typical dead cells by electron microscopy (2). In 1998, apoptosis was detected by the terminal deoxynucleotidyl transferase dUTP nick end labelling method in intervertebral discs. Excessive apoptosis causes the death of nucleus pulposus cells (3), and apoptosis is associated with the degeneration of cartilage endplates. In addition, the apoptotic rate has been demonstrated to increase under long-term pressure in intervertebral discs (4). Hence, we speculate that apoptosis is vital in intervertebral disc degeneration.

Apoptosis mediated by Fas (also known as CD95) or Fas ligand (FasL) is responsible for the loss of disc cells. High expression of Fas and FasL has been observed in intervertebral disc protrusions (5) and patients suffering from degenerative disc disease; however, age is insignificant with respect to the expression of FasL. Intervertebral disc cells negate FasL-mediated apoptosis under autocrine or paracrine Fas. Apoptosis and cell proliferation maintain relative balance, whereas the apoptotic rate is greater than the proliferation rate. The negative balance between the two processes causes cell shrinkage and disappearance, which is significant in the degeneration of intervertebral discs (6).

Following herniation, the disc cells undergo apoptosis through autocrine or paracrine FasL (7). Fas and FasL belong to the tumour necrosis factor family; their combination enables the Fas-FasL apoptotic pathway. Fas death receptor initiates apoptosis through the assemblages of Fas-associated death domain (FADD) and procaspase-8, subsequent proteolytic cleavage of vital structural substrates, and initiation of the caspase cascade (8). Caspase cascades, which lead to the activation of caspase- 3 , are essential in the process of apoptosis in the injured spinal cord (9). In this study, Fas and 
caspase-3 were examined in FasL-induced annulus fibrosus cells.

Paeonia lactiflora has been used in traditional Chinese prescriptions to treat certain types of nociceptive diseases, including muscle and menstrual pain (10-12). Paeoniflorin $(\mathrm{PF})$ is a bioactive glucoside isolated from the roots of P. lactiflora Pall. (13). PF exhibits biological and biomodulating activity, including memory improvement, as well as antioxidant and anti-inflammatory activity (14). PF also demonstrates cytoprotective effects on various cell types. PF inhibits $\mathrm{H}_{2} \mathrm{O}_{2}$-induced apoptosis by suppressing caspase-3 activity (15). However, the protective activity of PF in annulus fibrosus cells against FasL-induced apoptosis is yet to be elucidated. We postulated that PF mediates its effects through the modulations of the Fas-FasL signalling pathway and FasL-induced apoptosis.

\section{Materials and methods}

Culture and characterisation of annulus fibrosus cells. Sprague Dawley (SD) rats were anaesthetised with $10 \%$ chloral hydrate (3 $\mu \mathrm{l} / \mathrm{g})$. Disc fibre rings were isolated in a sterile manner, and superficial disc fibre rings were harvested and rinsed in $1 \mathrm{X}$ phosphate-buffered saline (PBS; Hyclone, Carlsbad, CA, USA) three times. The disc fibre rings were cut into sections (volume, $1 \mathrm{~mm}^{3}$ ). To isolate the cells, the disc tissues were digested with $0.25 \%$ trypsin including $0.02 \%$ ethylenediaminetetraacetic acid (Hyclone) for $20 \mathrm{~min}$, followed by another treatment with $0.2 \%$ collagenase II (Solarbio Science and Technology Co., Ltd., Beijing, China) for $8 \mathrm{~h}$ at $37^{\circ} \mathrm{C}$. Following the enzyme digestion, the suspension was filtered through a $70-\mu \mathrm{m}$ mesh. The filtered cells were harvested, and the digestion solution was refreshed twice. The cells were adjusted to a density of $2 \times 10^{5} / \mathrm{ml}$ by a hemocytometre; the cells were then placed in a humidified incubator with $5 \% \mathrm{CO}_{2}$ at $37^{\circ} \mathrm{C}$ in Dulbecco's modified Eagle's medium (DMEM) supplemented with $20 \%$ fetal bovine serum (FBS). The cells were passaged at $80 \%$ confluence, which was determined by microscopy.

Third-passage annulus fibrosus cells were plated onto coverslips, rinsed in 1X PBS, fixed in 4\% paraformaldehyde for $30 \mathrm{~min}$, and stained with $1 \%$ toluidine blue for $30 \mathrm{~min}$ at room temperature. The sections were briefly rinsed in absolute ethyl alcohol, dried, transparentised, mounted and observed via microscopy (original magnification, x200).

Animals. One-month-old male or female SD rats ( $\mathrm{n}=50$; weight, 100-120 g) were purchased from Slaccas Laboratory Animal Co., Ltd. (Shanghai, China; certification no. SCXX (SH) 2008-0005). The care and use of animals conformed to the regulations of the Guidelines for Laboratory Animal Welfare established by the Ministry of Science and Technology of China (16).

MTT assay. Third-passage annulus fibrosus cells were trypsinised and subcultured into 96 -well plates at $5 \times 10^{3}$ cells/well. Each group comprised five wells with an untreated group as a control. When the cells were anchored to the plates, various concentrations of PF (2080, 208, 20.8, 2.08, 0.208 and $0.0208 \mu \mathrm{M}$; National Institute for Control of Pharmaceutical and Biologic Products, Fujian, China) were incubated for 24,
48 and $72 \mathrm{~h}$. The activity of the annulus fibrosus cells was examined by 3-(4,5-dimethylthiazol-2-yl)-2,5-diphenyltetrazolium bromide (MTT) assay.

The cells were digested, centrifuged, collected and rinsed in 1X PBS. Twenty microlitres of $0.5 \%$ MTT (Solarbio Science and Technology Co., Ltd.) solution was added to each well and incubated at $37^{\circ} \mathrm{C}$ for $4 \mathrm{~h}$. MTT was discarded and replaced by $150 \mu 1$ dimethyl sulfoxide (Solarbio Science and Technology Co., Ltd.), and the mixture was blended for $10 \mathrm{~min}$. The optical density (OD) was determined for each group by a microplate reader (Thermo Scientific, Waltham, MA, USA) at $490 \mathrm{~nm}$; the mean values were calculated.

Flow cytometric analysis. Third-passage annulus fibrosus cells were trypsinised and subcultured into 6-well plates at $3 \times 10^{5}$ cells/well (final volume, $2 \mathrm{ml}$ ), and then incubated in DMEM with 20\% FBS (Hyclone). After reaching $80 \%$ confluence, the cells were freshly incubated in DMEM with $1 \%$ FBS (Hyclone) for $8 \mathrm{~h}$ (15) and induced with FasL (R\&D Systems, Minneapolis, MN, USA) at final concentrations of 0, 10, 20 and $40 \mathrm{ng} / \mathrm{ml}$ for $24 \mathrm{~h}$. The apoptotic rate was measured by flow cytometry using Annexin V-FITC/PI (Invitrogen, Carlsbad, CA, USA).

The cells were digested, centrifuged and rinsed in $1 \mathrm{X}$ PBS at $4^{\circ} \mathrm{C}$. The rinsed cells were resuspended with binding buffer at a density of $1 \times 10^{6} / \mathrm{ml}$. The cells were stained with $5 \mu 1$ Annexin V-FITC and $10 \mu \mathrm{l}$ PI in the dark at room temperature for $15 \mathrm{~min}$. A $400 \mathrm{ml}$ volume of the buffer was added to the resuspending cells; the cells were then sampled by flow cytometry (BD FACSCalibur, BD Biosciences, San Jose, CA, USA) to analyse the apoptotic fraction of the annulus fibrosus cells.

Western blot analysis. Third-passage annulus fibrosus cells were trypsinised and subcultured into 6 -well plates at $3 \times 10^{5}$ cells/well (final volume, $2 \mathrm{ml}$ ), and then incubated in DMEM with $20 \%$ FBS. After reaching $80 \%$ confluence, the cells were replaced by DMEM with $1 \%$ FBS for $8 \mathrm{~h}$ and induced with FasL at final concentrations of $20 \mathrm{ng} / \mathrm{ml}$; the cells were then treated with PF at final concentrations of 208, 20.8 and $2.08 \mu \mathrm{M}$ for $24 \mathrm{~h}$.

Total proteins were isolated from the cells; protein concentrations were tested by the bicinchoninic acid assay method (Sangon Biotech Co., Ltd., Shanghai, China). Samples of $40 \mu \mathrm{g}$ total protein were separated by gel electrophoresis using $12 \%$ SDS gel; the samples were then transferred to polyvinylidene difluoride (PVDF) membranes. The PVDF membrane was incubated with $1 \mathrm{X}$ Tris-buffered saline with $0.1 \%$ Tween-20 (TBST) and 5\% dehydrated skimmed milk to block non-specific protein binding for $2 \mathrm{~h}$; the proteins were incubated with rabbit anti-Fas (Cell Signaling Technology, Inc., Beverly, MA, USA).

Subsequently, 1:1,000 each of anti-caspase-3, anti-GAPDH, and anti- $\beta$-actin antibodies (all from Cell Signaling Technology, Inc.) were incubated overnight at $4^{\circ} \mathrm{C}$; anti-rabbit secondary antibodies (Cell Signaling Technology, Inc.) were incubated for $1 \mathrm{~h}$ at room temperature. The membranes were rinsed in TBST three times for $5 \mathrm{~min}$, labelled with enhanced chemiluminescence substrates, and exposed to X-ray. Protein bands were analysed using a gel imaging analysis system (Bio-Rad, Hercules, CA, USA) and normalised to $\beta$-actin in the sample. 

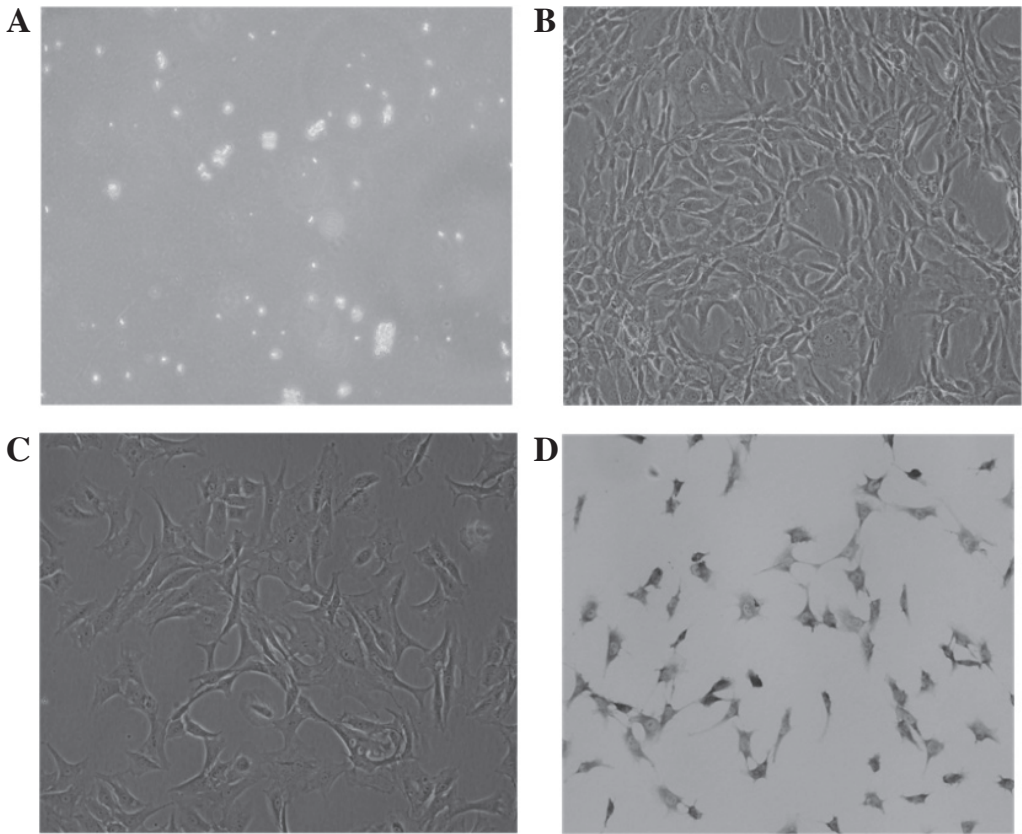

Figure 1. Morphology and characterisation of annulus fibrosus cells in vitro. (A) Primary digestion annulus fibrosus cells. (B) Primary culture on day 8 . (C) Third-passage culture on day 1. (D) Third-passage culture on day 1 stained with toluidine blue. Magnification, x200.

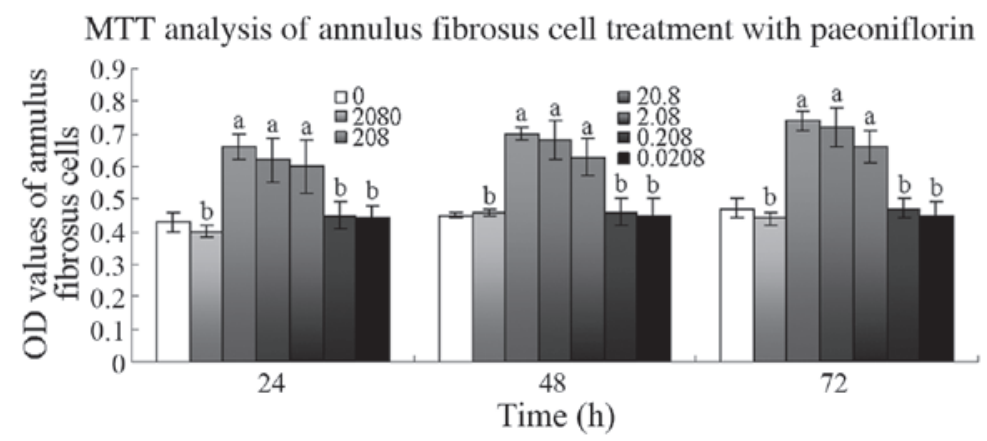

Figure 2. Effect of paeoniflorin (PF) on annulus fibrosus cell viability. Cells were treated with $0.0208,0.208,2.08,20.8,208$ and $2080 \mu \mathrm{M}$ PF for 24,48 and $72 \mathrm{~h}$, and cell viability was determined by 3-(4,5-dimethylthiazol-2-yl)-2,5-diphenyltetrazolium bromide (MTT) assay. ${ }^{a} \mathrm{P}<0.01$, ${ }^{\mathrm{b}} \mathrm{P}>0.05 \mathrm{vs} .0 \mu \mathrm{M}$ group. OD, optical density.

Statistical analysis. Data are expressed as the mean \pm standard deviation. The groups were compared by analysis of variance; $\mathrm{P}<0.05$ was considered to indicate a statistically significant difference.

\section{Results}

Morphology and characterisation of annulus fibrosus cells in vitro. The primary digestion annulus fibrosus cells were small and round; strong refraction was observed in the medium (Fig. 1A). On the first day of culture, the annulus fibrosus cells adhered to the culture flask and extended pseudopod-like projections; their nuclei were mainly round or oval. On the fourth day, the cells were mainly polygonal and in grouped distributions. The cells became confluent and arranged regularly on the eighth day (Fig. 1B). Second-, third- and fourth-passage cells grew rapidly; the passaged cells adhered to the culture flask within $12 \mathrm{~h}$. The period of subculture was 7 days; third-passage cells were mainly polygonal and all equal in size (Fig. 1C). The annulus fibrosus cells were detected by toluidine blue staining for characterisation. The third-passage annulus fibrosus cells were purple and exhibited metachromatic granules. The nuclei were mainly round or oval and dark blue (Fig. 1D).

MTT assay. The OD values were significantly higher in the cells treated with 208, 20.8 and $2.08 \mu \mathrm{M}$ PF after $24 \mathrm{~h}$ than those in untreated cells $(\mathrm{P}<0.01)$. At 48 and $72 \mathrm{~h}$, the $\mathrm{OD}$ values of the cells treated with $208,20.8$ or $2.08 \mu \mathrm{M}$ PF were also significantly higher than those of the untreated cells $(\mathrm{P}<0.01$; Fig. 2).

Inhibitory effects of PF treatment on FasL-induced apoptosis. Flow cytometry of annexin V-FITC/PI-stained cells was used to analyse the inhibitory effects of PF treatment on FasL-induced apoptosis in annulus fibrosus cells. The percentages of early and late apoptotic cells following treatment with $208,20.8$ or $2.08 \mu \mathrm{M}$ PF were significantly lower than those in untreated cells $(\mathrm{P}<0.01)$. The percentages of early and late apoptotic cells following treatment with $208 \mu \mathrm{M}$ PF were 
A

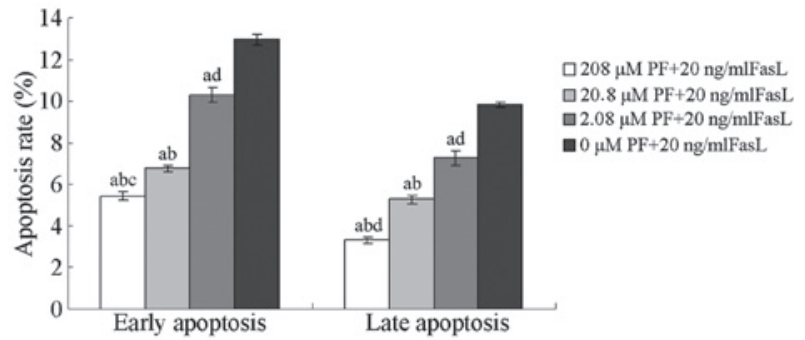

B
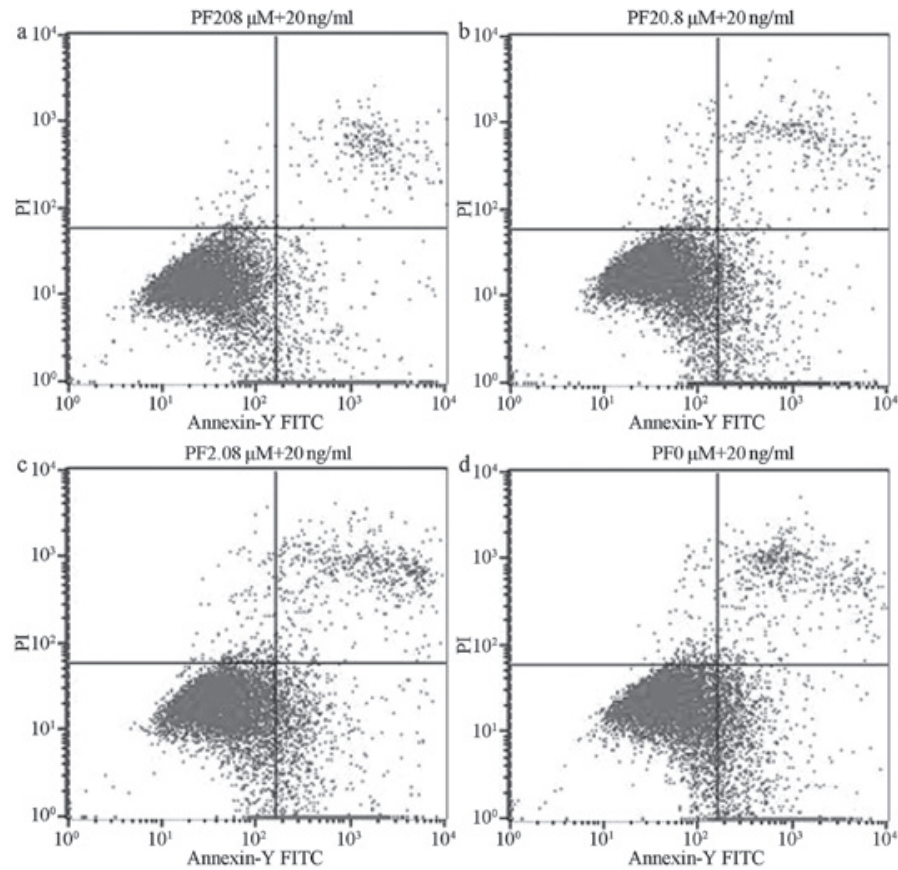

Figure 3. (A) Paeoniflorin (PF) treatment inhibits the apoptosis of annulus fibrosus cells induced by Fas ligand (FasL). ${ }^{\mathrm{a}} \mathrm{P}<0.01$ compared with $0 \mu \mathrm{M}$ $\mathrm{PF}+20 \mathrm{ng} / \mathrm{ml} \mathrm{FasL} ;{ }^{\mathrm{b}} \mathrm{P}<0.05$ vs. $2.08 \mu \mathrm{M} \mathrm{PF}+20 \mathrm{ng} / \mathrm{ml} \mathrm{FasL} ;{ }^{\mathrm{C}} \mathrm{P}>0.05,{ }^{\mathrm{d}} \mathrm{P}<0.05$ vs. $20.8 \mu \mathrm{M}$ PF $+20 \mathrm{ng} / \mathrm{ml}$ FasL. (B) PF treatment inhibits the apoptotic rate of annulus fibrosus cells induced by FasL. The percentage of early and late apoptotic cells was significantly lower when treated with $208,20.8$ or $2.08 \mu \mathrm{M}$ PF compared with untreated cells.

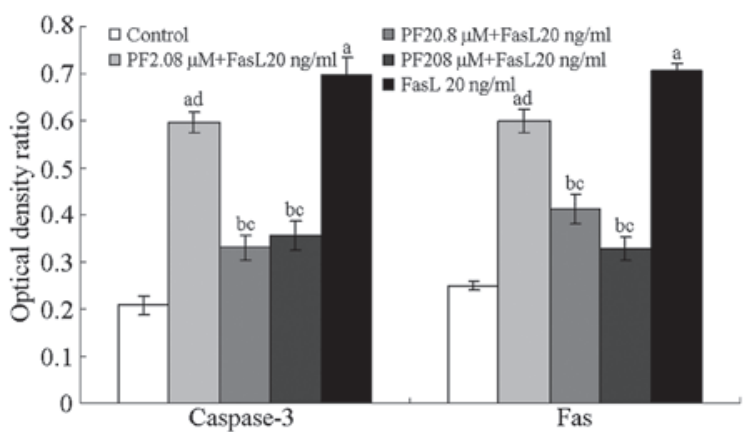

$\mathbf{B}$

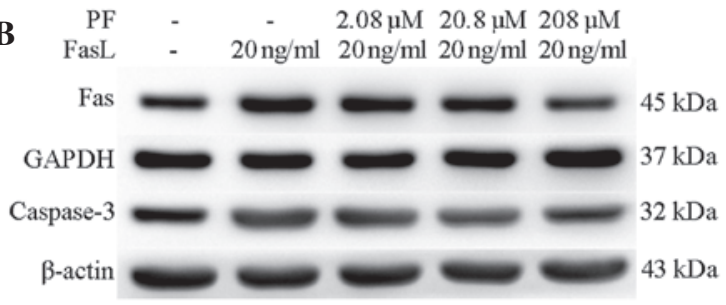

Figure 4. (A) Expression of Fas and caspase-3 in paeoniflorin (PF)-treated annulus fibrosus cells. ${ }^{a} \mathrm{P}<0.01,{ }^{b} \mathrm{P}<0.05$ vs. control group; ${ }^{\mathrm{C}} \mathrm{P}<0.01,{ }^{\mathrm{d}} \mathrm{P}<0.05$ vs. Fas ligand (FasL) $20 \mathrm{ng} / \mathrm{ml}$. (B) Expression levels of Fas and caspase-3 in PF-treated annulus fibrosus cells. PF treatment was given at 208, 20.8 or $2.08 \mu \mathrm{M}$ in FasL-induced $(20 \mathrm{ng} / \mathrm{ml})$ cells. The Fas and caspase- 3 expression levels significantly increased with FasL, and significantly decreased with PF treatment. lower than those of cells treated with $2.08 \mu \mathrm{M}$ PF ( $>>0.05)$. The percentage of late apoptotic cells was lower following treatment with $208 \mu \mathrm{M}$ PF than when treated with $20.8 \mu \mathrm{M}$ $\mathrm{PF}(\mathrm{P}>0.05)$; no significant differences were observed in the percentages of early apoptotic cells ( $\mathrm{P}>0.05$; Fig. 3 ).

Expression of Fas and caspase-3 in PF-treated annulus fibrosus cells. Following treatment with 208,20.8 and $2.08 \mu \mathrm{M}$ $\mathrm{PF}$, the annulus fibrosus cells demonstrated significantly lower levels of caspase- 3 protein than untreated cells $(\mathrm{P}<0.01)$; the protein levels in FasL-induced cells were higher than those in normal cells $(\mathrm{P}<0.05)$. Fas levels following 208, 20.8 and $2.08 \mu \mathrm{M}$ PF treatment were significantly lower than those in untreated cells $(\mathrm{P}<0.05)$; FasL-induced annulus fibrosus cells exhibited higher Fas levels compared with normal fibrosus cells $(\mathrm{P}<0.05$; Fig. 4).

\section{Discussion}

PF exhibits pharmacological activity, including antioxidant, anti-inflammatory and neuroprotective effects, on various types of cells (17-19). The protective activity of PF against FasL-induced injury was evaluated in annulus fibrosus cells. We demonstrated that $208,20.8$ or $2.08 \mu \mathrm{M}$ PF promoted the 
proliferation of annulus fibrosus cells and inhibited FasL-induced apoptosis of annulus fibrosus cells; the results were consistent with a previous study on the cytoprotective effects of PF (20).

The apoptotic rate of the annulus fibrosus cells was assessed following treatment with 10, 20 or $40 \mathrm{ng} / \mathrm{ml}$ FasL for $24 \mathrm{~h}$. The cells exhibited varying degrees of apoptosis; the apoptotic rates significantly increased with the dosage of FasL. The results revealed that the cells induced with $20 \mathrm{ng} / \mathrm{ml}$ FasL resulted in a moderate apoptotic rate, which was suitable for follow-up experiments (data not shown). Furthermore, $24 \mathrm{~h}$ FasL treatment effectively activated Fas-FasL signalling and resulted in the apoptosis of annulus fibrosus cells.

Fas, also known as CD95 and APO-1, is a widely studied receptor (21). When the Fas binds with its agonists, the death domain of intracellular gathered to trimer, then raise Fas-associated death domain (FADD) of cytoplasm. Then binds through its own death domain to the clustered receptor death domains. In addition, FADD contains a 'death effector domain' that binds to an analogous domain repeated in tandem within the zymogen form of caspase- 8 , which comprises Fas FADD procaspase 8 , referred to as the death-inducing signalling complex (DISC) (22). DISC activates the caspase-dependent apoptotic signalling cascade; the activation results in the cleavage of procaspase- 8 and -10 and caspase- 3 as well as the release of active caspase- 8 and -10 from the DISC (23). Caspase-3 initiates apoptosis by releasing caspase-activated deoxyribonuclease (CAD) from the inactive complex formed with the CAD inhibitor; CAD triggers the rapid fragmentation of DNA (24). Disc cells die during the rapid expression of Fas protein. Factors upregulated Fas in vivo and in vitro; enhancing the Fas-FasL system causes apoptosis. The breakage of the balance between cell proliferation and apoptosis reduces the disc cells. The change in matrix ingredients and the remodelling damages and destroys the physiological functions of intervertebral discs. This change is also referred to as organised intervertebral disc degeneration (25).

The results revealed that PF reduced the expression of Fas and caspase- 3 protein, which indicates that this compound reduced the percentage of dead fibre ring cells by the passageway of Fas-FasL signal transduction, which halted the regression of intervertebral discs.

\section{Acknowledgements}

This study was supported by the National Natural Science Foundation of China: Control system based on ontology sense discussed bilateral back for 'theory in the treatment of chronic lumbago role connotation' (no. 81303017) and the National 12th 'Five-Year' Technology Support Programs (no. 2013BAI10B05).

\section{References}

1. Ahmed SH, El-Shaarawy EA, Ishaq MF and Moniem MH: Morphological and radiometrical study of the human intervertebral foramina of the cervical spine. Folia Morphol (Warsz) 73: 7-18, 2014

2. Kong D, Zheng T, Fang J and Li X: Apoptosis of endplate chondrocytes in post-laminectomy cervical kyphotic deformity. An in vivo animal model in sheep. Eur Spine J 22: 1576-1582, 2013.

3. Jiang L, Zhang X, Zheng X, et al: Apoptosis, senescence, and autophagy in rat nucleus pulposus cells: Implications for diabetic intervertebral disc degeneration. J Orthop Res 31: 692-702, 2013
4. Chan SC, Ferguson SJ and Gantenbein-Ritter B: The effects of dynamic loading on the intervertebral disc. Eur Spine J 20: 1796-1812, 2011.

5. Ma KG, Shao ZW, Yang SH, et al: Autophagy is activated in compression-induced cell degeneration and is mediated by reactive oxygen species in nucleus pulposus cells exposed to compression. Osteoarthritis Cartilage 21: 2030-2038, 2013.

6. Guan YJ,Zhang Z, Yu C,MaL,Hu W and XuL:Phospho-SXXE/D motif mediated TNF receptor 1-TRADD death domain complex formation for T cell activation and migration. J Immunol 187: 1289-1297, 2011

7. Lee EW, Seo J, Jeong M, Lee S and Song J: The roles of FADD in extrinsic apoptosis and necroptosis. BMB Rep 45: 496-508, 2012.

8. Wang S, Xia P, Shi L and Fan Z: FADD cleavage by NK cell granzyme $M$ enhances its self-association to facilitate procaspase-8 recruitment for auto-processing leading to caspase cascade. Cell Death Differ 19: 605-615, 2012.

9. Sun Z, Wan ZY, Guo YS, Wang HQ and Luo ZJ: FasL on human nucleus pulposus cells prevents angiogenesis in the disc by inducing Fas-mediated apoptosis of vascular endothelial cells. Int J Clin Exp Pathol 6: 2376-2385, 2013.

10. Wang C, Yuan J, Wu HX, et al: Paeoniflorin inhibits inflammatory responses in mice with allergic contact dermatitis by regulating the balance between inflammatory and anti-inflammatory cytokines. Inflamm Res 62: 1035-1044, 2013.

11. Liu HQ, Zhang WY, Luo XT, Ye Y and Zhu XZ: Paeoniflorin attenuates neuroinflammation and dopaminergic neurodegeneration in the MPTP model of Parkinson's disease by activation of adenosine A1 receptor. Br J Pharmacol 148: 314-325, 2006.

12. Wang D, Wong HK, Feng YB and Zhang ZJ: Paeoniflorin, a natural neuroprotective agent, modulates multiple anti-apoptotic and pro-apoptotic pathways in differentiated PC12 cells. Cell Mol Neurobiol 33: 521-529, 2013.

13. Salunga TL, Tabuchi Y, Takasaki I, et al: Identification of genes responsive to paeoniflorin, a heat shock protein-inducing compound, in human leukemia U937 cells. Int J Hyperthermia 23: 529-537, 2007.

14. Wu D, Chen J, Zhu H, et al: UPLC-PDA determination of paeoniflorin in rat plasma following the oral administration of Radix Paeoniae Alba and its effects on rats with collagen-induced arthritis. Exp Ther Med 7: 209-217, 2014.

15. Wu YM, Jin R, Yang L, et al: Phosphatidylinositol 3 kinase/protein kinase $\mathrm{B}$ is responsible for the protection of paeoniflorin upon $\mathrm{H}_{2} \mathrm{O}_{2}$-induced neural progenitor cell injury. Neuroscience 14: 54-62, 2013.

16. The Ministry of Science and Technology of the People's Republic of China: Guidance Suggestions for the Care and Use of Laboratory Animals, 2006.

17. Liu ZH, Sun Z, Wang HQ, et al: FasL expression on human nucleus pulposus cells contributes to the immune privilege of intervertebral disc by interacting with immunocytes. Int J Med Sci 10: 1053-1060, 2013.

18. Ji Y, Wang T, Wei ZF, Lu GX, Jiang SD, Xia YF and Dai Y: Paeoniflorin, the main active constituent of Paeonia lactiflora roots, attenuates bleomycin-induced pulmonary fibrosis in mice by suppressing the synthesis of type I collagen. J Ethnopharmacol 149: 825-832, 2013.

19. Zhang MH, Feng L, Zhu MM, Gu JF, Wu C and Jia XB: Antioxidative and anti-inflammatory activities of paeoniflorin and oxypaeoniflora on AGEs-induced mesangial cell damage. Planta Med 79: 1319-1323, 2013.

20. Wankun X, Wenzhen Y, Min Z, et al: Protective effect of paeoniflorin against oxidative stress in human retinal pigment epithelium in vitro. Mol Vis 17: 3512-3522, 2011.

21. Lavrik IN and Krammer PH: Regulation of CD95/Fas signaling at the DISC. Cell Death Differ 19: 36-41, 2012.

22. Ashkenazi A and Dixit VM: Death Receptors: Signaling and modulation. Science 281 (5381): 1305-1308, 1998.

23. Park JB, Chang H and Kim KW: Expression of Fas ligand and apoptosis of disc cells in herniated lumbar disc tissue. Spine 26: 618-621, 2001.

24. Lechardeur D, Drzymala L, Sharma M, et al: Determinants of the nuclear localization of the heterodimeric DNA fragmentation factor (ICAD/CAD). J Cell Biol 150: 321-334, 2000.

25. Mehrkens A, Karim MZ, Kim S, Hilario R, Fehlings MG and Erwin WM: Canine notochordal cell-secreted factors protect murine and human nucleus pulposus cells from apoptosis by inhibition of activated caspase-9 and caspase-3/7. Evid Based Spine Care J 4: 154-156, 2013. 